\title{
Application of associating liver partition and portal vein ligation for staged hepatectomy for hepatocellular carcinoma related to hepatitis B virus: comparison with traditional one-stage right hepatectomy
}

\author{
Junwei Zhang ${ }^{1}$, Yiyao Xu ${ }^{2}$, Huayu Yang ${ }^{2}$, Hanchun Huang ${ }^{1}$, Jin Bian ${ }^{1}$, Shitao Jiang ${ }^{1}$, Xinting Sang ${ }^{2}$, \\ Yilei $\mathrm{Mao}^{2}$, Haitao $\mathrm{Zhao}^{2}$, Shunda $\mathrm{Du}^{2}$, Haifeng $\mathrm{Xu}^{2}$, Yongchang Zheng ${ }^{2}$, Tianyi Chi ${ }^{2}$, Xin $\mathrm{Lu}^{2}$ \\ ${ }^{1}$ Department of Liver Surgery, Peking Union Medical College (PUMC) Hospital, Chinese Academy of Medical Sciences and Peking Union Medical \\ College (CAMS \& PUMC), Beijing, China; ${ }^{2}$ Department of Liver Surgery, Peking Union Medical College (PUMC) Hospital, Chinese Academy of \\ Medical Sciences (CAMS), Beijing, China \\ Contributions: (I) Conception and design: J Zhang; (II) Administrative support: X Sang ; (III) Provision of study materials or patients: Y Mao, X Sang; \\ (IV) Collection and assembly of data: J Zhang, S Jiang, J Bian; (V) Data analysis and interpretation: H Huang; (VI) Manuscript writing: All authors; \\ (VII) Final approval of manuscript: All authors. \\ Correspondence to: Xin Lu, PhD. Department of Liver Surgery, Peking Union Medical College (PUMC) Hospital, PUMC \& Chinese Academy of \\ Medical Sciences (CAMS), Beijing 100730, China. Email: luxinln@163.com.
}

Background: Associating liver partition and portal vein ligation for staged hepatectomy (ALPPS) has recently been suggested to cause rapid liver hypertrophy among patients having inadequate future liver remnant (FLR). However, ALPPS is still considered as a controversial hepatocellular carcinoma (HCC) treatment, especially for those with cirrhosis. This is ascribed to the high mortality and morbidity. The present study aimed to evaluate the ALPPS safety and feasibility for HCC patients related to hepatitis B virus (HBV).

Methods: This was a retrospective observational study. Consecutive HCC cases undergoing ALPPS or RH at our hospital between September 2014 and June 2018 were included. The demographic and clinical data of patients were collected, and oncological results of ALPPS patients were compared with those receiving right hepatectomy $(\mathrm{RH})$.

Results: A total of 14 ALPPS patients and $21 \mathrm{RH}$ patients were consecutively collected between September 2014 and June 2018. All ALPPS patients received stage II operations, with $100 \%$ resection of R0. The median growth of FLR between operations was $48 \%$ (range, $-0.06 \%$ to $100 \%$ ) in 17 days (range, 9-30 days). 3 ALPPS patients (21.4\%) suffered from severe complications (grade $\geq \mathrm{IIIb}$ ) according to the Clavien-Dindo grade, including 1 with renal failure, and 2 with the FLR/SLV of $<30 \%$ and liver failure, and 1 (4.8\%) with severe complication (liver failure) after the stage I RH. Difference in the long-term survival, either overall survival (OS) or disease-free survival (DFS), between ALPPS and RH was not statistically significant.

Conclusions: Our results suggest that ALPPS should be performed in highly selected patients with cirrhosis. Patients with $30 \%<$ the FLR/SLV $<30 \%$ and ages $<60$ years old are recommended. And a longer interval time between stages is suggested to improve the safety of ALPPS. Because the long-term survival after ALPPS is similar to that after RH, which indicates that the long waiting time between stage does not increase the risk of tumor progression.

Keywords: Associating liver partition and portal vein ligation for staged hepatectomy (ALPPS); hepatocellular carcinoma (HCC); hepatitis B virus (HBV); survival analysis

Submitted Nov 28, 2019. Accepted for publication Jul 08, 2020.

doi: $10.21037 /$ tcr-19-2633

View this article at: http://dx.doi.org/10.21037/tcr-19-2633 


\section{Introduction}

Liver cancer ranks the sixth and fourth places in terms of its morbidity and mortality, respectively, worldwide (1). Surgical resection remains a major treatment for hepatocellular carcinoma (HCC) among cases with conserved hepatic functions $(2,3)$. However, many patients fail to receive surgical treatment due to inadequate future liver remnant (FLR), particularly for hepatitis B virus (HBV)-induced HCC patients receiving right hepatectomy (RH) (4).

At present, associating liver partition and portal vein ligation for staged hepatectomy (ALPPS) is recognized as a potent treatment for improving resectability in comparison with the traditional two-stage hepatectomy for cases developing liver metastases of advanced colorectal cancer (CRC) $(5,6)$.

Recently, ALPPS is introduced as the viable therapeutic choice among unresectable HCC patients with concomitant $\mathrm{HBV}$ infection (7-11). However, it is controversial to perform ALPPS in cases with concurrent HBV infection, since HCC usually occurs with cirrhosis, which restricts liver growth (12). Although radiofrequency (RF)-assisted ALPPS and tourniquet ALPPS have been employed to improve the safety of ALPPS for HCC, the 90-day mortality remains high, and $20 \%$ patients can't proceed to do the second stage of ALPPS $(8,9)$. Besides, the safety and feasibility of ALPPS for HCC patients remain questionable (13).

Studies show that the stricter selection criteria help to reduce hepatic failure following ALPPS $(14,15)$. However, no favorable benchmark is available to decide whether a HCC patient is eligible for ALPPS or not. Moreover, data on the long-term survival of HCC patients after ALPPS are lacking so far.

Therefore, this study was carried out aiming to assess the safety and efficacy of ALPPS for HBV-related HCC patients, and to explore a new eligibility standard. Besides, the oncologic long-term outcomes of ALPPS were compared with those of one-stage extended $\mathrm{RH}$.

We present the following article in accordance with the STROBE reporting checklist (available at http://dx.doi. org/10.21037/tcr-19-2633).

\section{Methods}

\section{Patients and study design}

This was a retrospective observational study. Between September 2014 and June 2018, consecutive HBV-related HCC cases undergoing ALPPS, together with HBV-related
HCC cases receiving RH, were enrolled from the Peking Union Medical College Hospital (PUMCH) into this study. We followed the Declaration of Helsinki (as revised in 2013), and the study was approved by the institutional ethics board of PUMCH (No: S-K1150). Informed consent was obtained from all patients. All cases were positive for HBV surface antigen and were diagnosed with HCC in line with CT or MRI findings. After the diagnosis of HCC, the treatment for patients was discussed by the multidisciplinary team, including liver surgery department, oncology department, radiotherapy department, and interventional department. Physicians recommended treatments for patients, but the final decision was made by patients themselves. For HCC patients undergoing RH, the liver remnant/standard liver volume (SLV) was judged by two experienced surgeons, respectively, and both of them were deemed as $>40 \%$. For HCC patients receiving ALPPS, the liver remnant/SLV was calculated based on CT scans, both of which were deemed as $<40 \%$.

Moreover, the King's scores, Child-Pugh scores, and MELD scores were also utilized to rate liver fibrosis in predicting the 1-year survival and 3-month mortality (16). In addition, the Couinaud's classification and the Brisbane 2000 Terminology of Liver Anatomy and Resections were utilized for surgical and anatomic designations (17). The International Study Group of Liver Surgery (ISGLS) classification was employed to grade the post-hepatectomy liver failure (PHLF) (18). Besides, postoperative complications (POC) were defined in accordance with the Clavien-Dindo standards, among which, grades IIIb and above were deemed as serious (19). Tumor relapse was diagnosed according to either histological evidence or noninvasive HCC diagnostic standards.

\section{Study size and potential biases}

We retrospectively collected patients who underwent ALPPS and RH between September 2014 and June 2018, and 14 ALPPS and 21 RH patients were enrolled finally. There were potential biases in this study due to its retrospective nature. Firstly, ALPPS was the two-stage liver resection, while RH was the one-stage liver resection, therefore, patients who underwent ALPPS certainly had more injury than RH. As a result, we only compared the operation time of stage I ALPPS with RH to balance the bias. Secondly, ALPPS and RH were performed by the surgical team at the same time, and their therapeutic schedule was given by the same team, which balanced the 
potential bias caused by the different surgical skills and therapy after surgery.

\section{Surgical procedure}

The ALPPS surgical technique in our hospital have been previously described, which is similar to the technique described in other publications (9). At the first stage, a Y-shaped incision was made in the upper abdomen, and then the abdominal cavity was explored to exclude the presence of any metastasis. In addition, ultrasonography was carried out intraoperatively to verify the relationship between hepatic vein and tumor. This ascertained whether the middle hepatic vein was completely preserved. The gallbladder was removed routinely, and the first hepatic hilum was dissected. Later, the right-sided portal vein was dissected, while the right hepatic artery and biliary duct must be carefully divided and preserved. Thereafter, the portal vein in the right side was cut to reveal the hepatic ischemic boundary in the right side. Such boundary, together with the middle hepatic vein position, was utilized to determine the detachment surface, and tissue was removed from the liver at the direction of hepatic tract. Subsequently, the bilaterally-dissected sections were treated one by one until the anterior wall of posterior inferior vena cava was completely exposed. Notably, it was unnecessary to dissect the right hepatic vein. Additionally, any active hemorrhage and bile leakage in the liver section were carefully examined. Moreover, a broad flap was used to mark the right hepatic duct and the right hepatic artery, and a drainage tube was placed between these two incisions under the liver.

At the second stage, right hepatic duct and right hepatic artery were identified and sutured in surgical markers, and the right hepatic ligament was severed. Then, the second hepatic hilum was dissociated, right hepatic vein was dissected, and the right liver was completely removed.

\section{Follow-up}

Once discharged, patients were followed up at our outpatient clinic, and digital subtraction angiography (DSA) was performed for twice at an interval of 4 weeks. Tumor recurrence or subclinical recurrence was suspected if any abnormal contrast medium concentration was detected in the liver. Enhanced CT and peripheral blood tumor markers were examined at 6 months after surgery if no abnormality was detected.

\section{Hepatic volumetry}

Hepatic volumetry was performed prior to and following the stage I ALPPS under the hand of the same radiologist certified by the board. Liver volume was calculated from images at the portal venous phase. The major branches of portal vein within liver hilum were carefully excluded. Afterwards, the resection plane was plotted under the assistance of surgeon, so as to automatically calculate the predicted FLR. When evaluating the eligibility of the increased FLR for stage-II resection, the estimated SLV was calculated according to the formula $\left[\operatorname{SLV}\left(\mathrm{cm}^{3}\right)=758.259\right.$ $\left.\times \operatorname{BSA}\left(\mathrm{m}^{2}\right)-124.272\right]$ for predicting SLV in Chinese adults (20). In HCC patients with a history of fibrosis or cirrhosis, a FLR/ SLV ratio of $>40 \%$ was considered as safe for proceeding to the stage-II operation. The kinetic growth rates (KGR), which reflect the weekly increase in the FLR volume, were calculated. We also referred to the liver transplantation standards, and an FLR and weight ratio of $>0.8 \%$ were regarded as adequate for the patient.

\section{Statistics}

The IBM SPSS Statistics 23.0 (SPSS, Chicago, IL, USA) was employed for all statistical analyses. Kolmogorovsmirnov test was used to test the data distribution. Data were expressed as mean standard deviation (SD) or median with ranges for normally or non-normally distributed data. Continuous data were presented as median, which were analyzed through student's T test or U test. And fisher exact test was used to compare the categorical data. Postoperative morbidity and mortality were graded according to the Clavien classification. The Kaplan-Meier approaches were utilized for survival analyses, while log-rank test was carried out for comparison. A difference of $\mathrm{P}<0.05$ indicated statistical significance. If the patient was lost to our followup, the last follow-up was deemed as the time that he quit the study. Clinical factors favoring the DFS and OS survival were identified using logistic regression.

\section{Results}

\section{Characteristics of patients and diseases}

From September 2014 and June 2018, a total of 35 HCC patients related to $\mathrm{HBV}$-virus underwent ALPPS $(\mathrm{n}=14)$ or RH ( $\mathrm{n}=21)$ at the Department of Liver surgery, Pecking Union medical College Hospital. Table 1 presents the basic demographic of patients and disease features. Differences in 
Table 1 Baseline characteristics of ALPPS and RH patients

\begin{tabular}{|c|c|c|c|}
\hline Characteristics & ALPPS $(n=14)$ & $\mathrm{RH}(\mathrm{n}=21)$ & $\mathrm{P}$ \\
\hline Male sex, (\%) & 78.6 & 81 & 0.594 \\
\hline BMI & $22.1 \pm 0.8$ & $23.3 \pm 1.6$ & 0.17 \\
\hline More than one tumor & 0 & 2 & 0.353 \\
\hline Diabetes (\%) & 0 & 4.8 & 0.6 \\
\hline Hypertension (\%) & 7.1 & 9.5 & 0.652 \\
\hline \multicolumn{4}{|l|}{ Blood value } \\
\hline Hemoglobin (g/L) & $137.4 \pm 6.5$ & $136.5 \pm 12.2$ & 0.90 \\
\hline INR & $1.1 \pm 0.03$ & $1.1 \pm 0.04$ & 0.81 \\
\hline Albumin (g/L) & $37 \pm 5.5$ & $40 \pm 2.15$ & 0.23 \\
\hline Bilirubin (mmol/L) & $16.3 \pm 3.7$ & $14.5 \pm 2.5$ & 0.42 \\
\hline Creatinine $(\mathrm{mmol} / \mathrm{L})$ & $68 \pm 5$ & $68 \pm 8$ & 0.93 \\
\hline MELD score & $4.3 \pm 1$ & $3.8 \pm 1.5$ & 0.61 \\
\hline Kings' score & $29.9 \pm 12.9$ & $17.8 \pm 6.1$ & 0.12 \\
\hline Child-Pugh grade & $5.1 \pm 0.18$ & $5.0 \pm 0.1$ & 0.34 \\
\hline
\end{tabular}

ALPPS, associating liver partition and portal vein ligation for staged hepatectomy; $\mathrm{RH}$, right hepatectomy; BMI, body mass index; MELD, model for end-stage liver disease; King's score, agex aspartate aminotransferase $\times$ international normalized ratio/platelet count.

the demographic data between ALPPS and RH cases were not statistically significant. All the 14 cases receiving ALPPS in the study period had a single tumor, and the median diameter was $10.8 \mathrm{~cm}$ (range, 7.7-14.1 cm). Among the 21 cases receiving RH surgery, 19 had a single tumor, with the median diameter of $9.4 \mathrm{~cm}$ (range, $2.2-16 \mathrm{~cm}$ ). Meanwhile, the median age was 54.8 years (range, 34-68 years) for ALPPS patients, and 49.4 years (range, 32-66 years) for RH patients. Differences in the blood tests between ALPPS and $\mathrm{RH}$ patients were not statistically significant, indicating the presence of the same degree of cirrhosis.

\section{Intraoperative data}

The average blood loss at stage I ALPPS was $432 \mathrm{~mL}$ (range, $300-1,200 \mathrm{~mL}$ ), while that of RH was $890 \mathrm{~mL}$ (range, 200$3,000 \mathrm{~mL}$ ). Difference was statistically significant between both groups $(\mathrm{P}=0.025)$. The average blood transfusion was $2.3 \mathrm{U}$ red cells (range, $0-8 \mathrm{U}$ ) in stage I ALLPS, while that was 2.9 $\mathrm{U}$ red cell (range, $0-8 \mathrm{U}$ ) in RH. All ALLPS patients underwent complete surgical resection and negative margins were achieved. The average operation time of stage I ALPPS was $248.3 \mathrm{~min}$ (range, 169-373 $\mathrm{min}$ ), while that in RH procedures was $241.6 \mathrm{~min}$ (range, $177-409 \mathrm{~min}$ ). The complete resection (R0) rate of ALPPS was 100\% (Table 2).

\section{Postoperative morbidity and mortality}

All patients proceeded to stage II operation. The incidence of severe complications was $21.4 \%$ in ALPPS. One patient suffered from renal failure after stage I operation and recovered very well after dialysis. Two patients experienced PHLF after stage II operation, which was rated as a grade C hepatic failure in accordance with the ISGLS (18). These two patients died within 2 months after discharge. The FLR/SLV of them were $<30 \%$ and the FLR/weights were $<0.6 \%$. One patient experienced severe complication (hepatic failure) after $\mathrm{RH}$ and died at two weeks after $\mathrm{RH}$ 
Table 2 Surgical outcome (ALPPS first stage compared with RH)

\begin{tabular}{lccc}
\hline Variable & ALPPS I & RH & P \\
\hline blood loss $(\mathrm{mL})$ & $432(100-1,200)$ & $890(200-3,000)$ & 0.025 \\
blood transfusion $(\mathrm{U})$ & $2.3(0-8)$ & $2.9(0-8)$ & 0.354 \\
Surgical time $(\mathrm{min})$ & $248.3(169-373)$ & $241.6(177-409)$ & 0.305 \\
Ro resection rate & $100 \%$ & $95.2 \% *$ & \\
\hline
\end{tabular}

*, one patient after RH was found to have tumors in the cutting edge under microscope, which was deemed as R1 resection. ALPPS I, The first stage of associating liver partition and portal vein ligation for staged hepatectomy; $\mathrm{RH}$, right hepatectomy.

Table 3 Postoperative Complications in Patients Undergoing ALPPS and RH

\begin{tabular}{|c|c|c|c|}
\hline & ALPPS $1(n=14)$ & ALPPS $2(n=14)$ & $\mathrm{RH}(\mathrm{n}=21)$ \\
\hline \multirow[t]{2}{*}{ Grade II } & Mild ascites, relieved by diuretics $(n=2)$ & Mild ascites, relieved by diuretics $(n=1)$ & Mild ascites, relieved by diuretics $(n=2)$ \\
\hline & $\begin{array}{l}\text { Gross ascites, not relieved by diuretics } \\
(n=1)\end{array}$ & Gross ascites, not relieved by diuretics $(n=1)$ & \\
\hline Grade IV & $\begin{array}{l}\text { Acute renal failure requiring dialysis } \\
(n=1)\end{array}$ & & \\
\hline Grade V & & $\begin{array}{l}\text { Hemorrhage and liver failure }(n=1) \text {; liver failure } \\
(n=1)\end{array}$ & \\
\hline Grade C & 0 & 2 & 0 \\
\hline
\end{tabular}

ALPPS, associating liver partition and portal vein ligation for staged hepatectomy; $\mathrm{RH}$, right hepatectomy.

(Table 3). The total length of hospital stay of ALPPS was 38.5 days (range, 25-54 days), which was significantly longer than the 17.5 days of RH (range, 14-21 days). Only one patient after ALPPS stayed at the intensive care unit (ICU) for 4 days after ALPPS because of renal failure, while three patients stayed at the ICU for one day after RH. The 30-days mortality was $0 \%$ for ALPPS and $4.7 \%$ for RH. However, the 90 -day mortality was $14 \%$ for ALPPS and $4.7 \%$ for $\mathrm{RH}$, which has no statistical difference $(\mathrm{P}=0.551$, fisher exact test)

\section{Volumetric changes in the liver}

The median preoperative FLR volume was $437 \mathrm{~mL}$ (range, 342-511 mL), which increased to $649 \mathrm{~mL}$ (range, $460-810 \mathrm{~mL}$ ) before stage I operation, resulting in the median increase by $212 \mathrm{~mL}$ (range, -30-362 mL). This was corresponding to a median increase by $48 \%$ (range, -0.06-100\%). The median preoperative FLR was 36\% (range, 27-44\%), which increased to 52\% (range, 36-72\%) before stage II ALPPS. The preoperative median FLR/ weight was $0.66 \%$ (range, $0.48-0.88 \%$ ), which increased to 0.95 (range, 0.65-1.26) before stage II ALPPS. The median interval between two operations was 17 days (range, 9-30 days). In addition, the absolute and relative KGR were $13.2 \mathrm{~mL} /$ day (range, $-1.3-22.5 \mathrm{~mL} /$ day) and 1.8\%/day (range, $-0.7-3.4 \% /$ day), respectively (Table 4).

Two patients had insufficient hypertrophy before stage II operation. One patient received ALPPS and had negative hypertrophy at 21 days after stage I operation. We performed stage II operation in case of liver infection and necrosis. After the second stage operation, the patient 
Table 4 The postoperative data of ALPPS patients

\begin{tabular}{lc}
\hline Variable & Median range \\
\hline Interval between stage-1 and stage-2 & $17.3(9-31)$ \\
SLV (Urata formula), mL & $1,192.8(1,050-1,340)$ \\
Before stage-1 operation & $437(342-511)$ \\
FLR, mL & $36(27-44)$ \\
FLR/SLV (\%) & $0.66(0.48-0.88)$ \\
FLR/weight (\%) & $649(460-810)$ \\
Before stage-2 operation & $52(36-72)$ \\
FLR, mL & $0.95(0.62-1.26)$ \\
FLR/SLV (\%) & \\
FLR/weight (\%) & $13.2(-1.3-23.5)$ \\
FLR increase before stage-2 operation (\%) \\
Absolute KGR, mL/day \\
Relative KGR, \%/day & $1.8(-0.8-3.4)$ \\
\hline
\end{tabular}

ALPPS, associating liver partition and portal vein ligation for staged hepatectomy; SLV, standard liver volume; FLR, future liver remnant; KGR, kinetic growth rate.

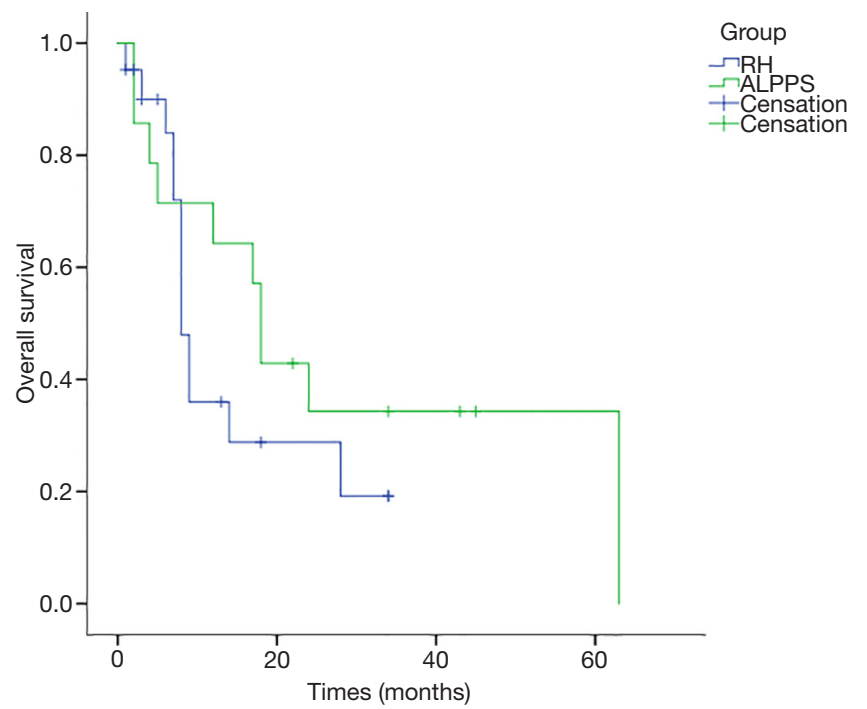

Figure 1 The OS of patients after ALPPS was similar to those with RH ( $\mathrm{P}=0.34, \mathrm{Log}$ Rank test). OS, overall survival; ALPPS, associating liver partition and portal vein ligation for staged hepatectomy; RH, right hepatectomy.

experienced fast liver growth from $460 \mathrm{~mL}(36.2 \%)$ to $576 \mathrm{~mL}$ (45.2\%). Another patient had hemorrhage after surgery, which aggravated hepatic failure and caused hepatic encephalopathy. Therefore, the stage II operation was performed in 20 days after the first stage.

\section{Follow up}

All cases were followed up for 15 months on average (range, 1-63 months). The 1-year OS rates were $64.3 \%$ for the 14 patients receiving ALPPS and 36\% for RH patients, and the 3-year OS rates were $34.3 \%$ for ALPPS and 19.2\% for RH patients; while the 1-year DFS rates were $32.7 \%$ for ALPPS patients and $36.3 \%$ for RH cases. According to $\mathrm{K}-\mathrm{M}$ analysis, the OS for patients undergoing ALPPS was similar to that of patients following one-stage liver resection $(\mathrm{P}=0.34)$, and the DFS was also equivalent $(\mathrm{P}=0.754)$ (Figures 1,2). And no clinical factors favors the DFS and OS survival were founded using logistic regression.

\section{Discussion}

ALPPS is considered as the potential solution for patients with liver cancer suffering from inadequate FLR. However, ALLPS is still controversial, though it has been practiced for about ten years (21-24). The safety of ALPPS is the highest concern since its first description by Schnitzbauer in 2012 (25). The selection of patients, surgical technique modification, and second stage timing can be used to improve the safety of ALPPS.

Our results suggest that ALPPS should be performed in highly selected patients with HBV-related HCC. $30 \%<$ the FLR/SLV $<30 \%$ and the ages $>60$ years old are recommended. And a longer interval time between stages is suggested to improve the safety of ALPPS. The longterm survival after ALPPS is similar to that after RH, which indicates that the long waiting time between stage does not increase the risk of tumor progression.

ALPPS has been proved in clinical trials and metaanalysis to achieve satisfactory hypertrophy for liver metastasis of advanced CRC (26,27). However, the safety and patient selection for ALPPS, especially for HCC patients, should be further investigated. The greatest concerns about the application of ALPPS among HCC patients are displayed as follows: (I) whether FLR can rapidly increase among liver cirrhosis patients within a short time and whether it is sufficient to compensate for liver function; (II) whether patients waiting for liver regeneration are prone to metastasis and have increased risks of recurrence; (III) whether it is worth performing ALPPS as a result of the increased complication risks, especially for bile 


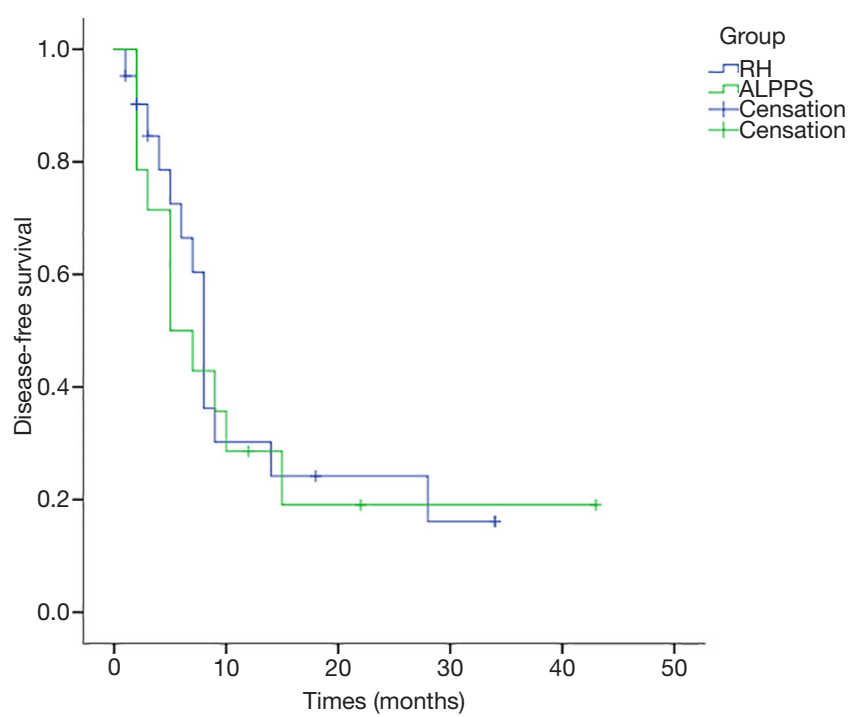

Figure 2 The DFS of patients after ALPPS was similar to those with $\mathrm{RH}$ ( $\mathrm{P}=0.754$, Log Rank test). DFS, disease-free survival; ALPPS, associating liver partition and portal vein ligation for staged hepatectomy; $\mathrm{RH}$, right hepatectomy.

leakage.

In the present study, all of our 14 patients successfully proceeded to the stage II operation, suggesting that cirrhotic liver regenerated quickly. Also, the stricter selection criteria, including FLR/SLV and FLR/weight, were applied for measurements. However, two patients had insufficient FLR when they underwent stage II surgery, including one because of the short interval time between stages (the patient underwent the stage II operation at 20 days after stage I one). The patient had fast liver regeneration just at 1 week after stage II operation. We assumed that liver with cirrhosis grew slower than the normal liver, as proved by previous studies $(28,29)$. Another patient was due to hemorrhage and liver failure after stage I operation, and we had to perform the stage II operation in advance. The patient died at two months after discharge, which indicated that it was not safe to perform the stage II operation if the stage I operation was performed ahead of plan. In this regard, we recommended a longer interval between stages to wait for liver hypertrophy. And if the FLV can't grow big enough, do not perform the second stage operation.

As for the short-term outcomes in this study, two ALPPS and one RH patients experienced PHLF after stage II operation. Both of the two patients after ALPPS had low FLR/SLV and FLR/weight ratios, and older than 60 years old; therefore, it was plausible that we should raise the criteria for HBV-related HCC. Typically, 40\%> FLR/SLV $>30 \%$ and ages $>60$ years old might serve as the favorable indications for HCC patients. One patient experienced renal failure after ALPPS, who recovered well after dialysis. Previous studies show that, patients with cirrhosis are more likely to develop renal failure, and its mechanism needs to be further studied (30).

Bile leakage is the common complication of ALPPS, which results in sepsis and hepatic failure (23). As a result, doctors have to perform the stage II operation in advance. Different methods have been employed to decrease the bile leakage rate, including plastic bags, tourniquets and radiofrequency-assisted ALPPS (8,9,31). However, these methods may introduce further complications, such as infection and slow growth rates. In our study, we observed no bile leakage in our patients, which suggested that using cavitron ultrasonic dissector to identify and ligate individual bile ducts during surgery were the important steps to reduce the bile leakage rate, and that it was useful to perform "delayed ALPPS" (22).

With regard to the long-term outcomes, this study showed that the OS and DFS for patients receiving ALPPS were similar to those undergoing RH. Such results demonstrated that advanced HCC patients might benefit from ALPPS, although they were associated with a higher risk during stage II operation, which further indicated that ALPPS should be performed successfully at an experienced center in highly selected patients.

The strengths of our study were that, all surgeries were performed by the same surgical team during the same period, which contributed to balancing numerous biases. Nonetheless, some limitations should be noted in our study. Firstly, this was an observational study, which might inevitably lead to recall bias. Also, some other factors not analyzed in subgroup due to the small sample size. Due to the small number of patients from one single center in our study, the results of our study should be interpreted with caution.

In conclusion, our study shows that ALPPS can be performed in highly selected patients at experienced centers in treating HBV-related HCC patients and that "delayed ALPPS" can reduce liver failure in patients receiving ALPPS, without inducing tumor progression.

\section{Acknowledgments}

Funding: This work was supported by the International Science and Technology Cooperation Projects 
(2016YFE0107100), the Capital Special Research Project for Health Development (2014-2-4012), the Beijing Natural Science Foundation (L172055), the National Ten-thousand Talent Program, the Fundamental Research Funds for the Central Universities (3332018032), and the CAMS Innovation Fund for Medical Science (CIFMS) (2017-I2M4-003).

\section{Footnote}

Reporting Checklist: The authors have completed the STROBE reporting checklist. Available at http://dx.doi. org/10.21037/tcr-19-2633

Data Sharing Statement: Available at http://dx.doi. org/10.21037/tcr-19-2633

Conflicts of Interest: All authors have completed the ICMJE uniform disclosure form (available at http://dx.doi. org/10.21037/tcr-19-2633). The authors have no conflicts of interest to declare.

Ethical Statement: The authors are accountable for all aspects of the work in ensuring that questions related to the accuracy or integrity of any part of the work are appropriately investigated and resolved. The study followed the Declaration of Helsinki (as revised in 2013), and the study was approved by the institutional ethics board of PUMCH (NO: S-K1150). Informed consent was obtained from all patients.

Open Access Statement: This is an Open Access article distributed in accordance with the Creative Commons Attribution-NonCommercial-NoDerivs 4.0 International License (CC BY-NC-ND 4.0), which permits the noncommercial replication and distribution of the article with the strict proviso that no changes or edits are made and the original work is properly cited (including links to both the formal publication through the relevant DOI and the license). See: https://creativecommons.org/licenses/by-nc-nd/4.0/.

\section{References}

1. Bray F, Ferlay J, Soerjomataram I, et al. Global Cancer Statistics 2018: GLOBOCAN Estimates of Incidence and Mortality Worldwide for 36 Cancers in 185 Countries. CA Cancer J Clin 2018;68:394-424.

2. Liu CY, Chen KF, Chen PJ. Treatment of Liver Cancer.
Cold Spring Harb Perspect Med 2015;5:a021535.

3. Agrawal S, Belghiti J. Oncologic resection for malignant tumors of the liver. Ann Surg 2011;253:656-65.

4. Kishi Y, Abdalla EK, Chun YS, et al. Three hundred and one consecutive extended right hepatectomies: evaluation of outcome based on systematic liver volumetry. Ann Surg 2009;250:540-8.

5. Sandström P, Rosok BI, Sparrelid E, et al. ALPPS Improves Resectability Compared With Conventional Two-stage Hepatectomy in Patients With Advanced Colorectal Liver Metastasis Results From a Scandinavian Multicenter Randomized Controlled Trial (LIGRO Trial). Ann Surg 2018;267:833-40.

6. Sparrelid E, van Gulik TM. Tumor progression in twostage liver resections-is the shorter inter-stage period in associated liver partition and portal vein ligation for staged hepatectomy (ALPPS) of benefit to the patient? Hepatobiliary Surg Nutr 2019;8:316-7.

7. Wang Z, Peng Y, Hu J, et al. Associating Liver Partition and Portal Vein Ligation for Staged Hepatectomy for Unresectable Hepatitis B Virus-related Hepatocellular Carcinoma. Ann Surg 2020;271:534-41.

8. Cai X, Tong Y, Yu H, et al. The ALPPS in the Treatment of Hepatitis B-Related Hepatocellular Carcinoma With Cirrhosis: A Single-Center Study and Literature Review. Surg Innov 2017;24:358-64.

9. Wang Q, Yan J, Feng X, et al. Safety and efficacy of radiofrequency-assisted ALPPS (RALPPS) in patients with cirrhosis-related hepatocellular carcinoma. Int J Hyperthermia 2017;33:846-52.

10. Xu YY, Lu X, Yang HY, et al. Application of associating liver partition and portal vein ligation for staged hepatectomy(ALPPS)in cirrhotic patients with hepatocellular carcinoma. Chinese Journal of General Surgery 2016;31:534-7.

11. Lau WY. Associating liver partition and portal vein ligation for staged hepatectomy (ALPPS) and its further developments in the last decade. Hepatobiliary Surg Nutr 2019;8:258-9.

12. Truant $\mathrm{S}$, Pruvot FR, Felli E, et al. Is there still a room to improve the safety of ALPPS procedure? A new technical note. Eur J Surg Oncol 2015;41:1554-5.

13. Moris D, Ronnekleiv-Kelly S, Kostakis ID, et al. Operative Results and Oncologic Outcomes of Associating Liver Partition and Portal Vein Ligation for Staged Hepatectomy (ALPPS) Versus Two-Stage Hepatectomy (TSH) in Patients with Unresectable Colorectal Liver Metastases: A Systematic Review and Meta-Analysis. 
World J Surg 2018;42:806-15.

14. Linecker M, Bjornsson B, Stavrou GA, et al. Risk Adjustment in ALPPS Is Associated With a Dramatic Decrease in Early Mortality and Morbidity. Ann Surg 2017;266:779-86.

15. Linecker M, Stavrou GA, Oldhafer KJ, et al. The ALPPS Risk Score: Avoiding Futile Use of ALPPS. Ann Surg 2016;264:763-71.

16. Pang Q, Bi JB, Xu XS, et al. King's score as a novel prognostic model for patients with hepatitis B-associated hepatocellular carcinoma. Eur J Gastroenterol Hepatol 2015;27:1337-46.

17. Pang YY. The Brisbane 2000 terminology of liver anatomy and resections. HPB 2000; 2:333-39. HPB (Oxford) 2002;4:99-100; author reply 99-100.

18. Rahbari NN, Garden OJ, Padbury R, et al. Posthepatectomy liver failure: a definition and grading by the International Study Group of Liver Surgery (ISGLS). Surgery 2011;149:713-24.

19. Dindo D, Demartines N, Clavien PA. Classification of surgical complications: a new proposal with evaluation in a cohort of 6336 patients and results of a survey. Ann Surg 2004;240:205-13.

20. Feng LM, Wang PQ, Yu H, et al. New formula for predicting standard liver volume in Chinese adults. World J Gastroenterol 2017;23:4968-77.

21. Olthof PB, Schnitzbauer AA, Schadde E. The HPB controversy of the decade: 2007-2017 - Ten years of ALPPS. Eur J Surg Oncol 2018;44:1624-7.

22. Lodge JP. ALPPS: The argument for. Eur J Surg Oncol 2017;43:246-8.

23. Wigmore SJ. ALPPS: The argument against. Eur J Surg Oncol 2017;43:249-51.

Cite this article as: Zhang J, Xu Y, Yang H, Huang H, Bian J, Jiang S, Sang X, Mao Y, Zhao H, Du S, Xu H, Zheng Y, Chi T, $\mathrm{Lu} X$. Application of associating liver partition and portal vein ligation for staged hepatectomy for hepatocellular carcinoma related to hepatitis B virus: comparison with traditional onestage right hepatectomy. Transl Cancer Res 2020;9(9):5371-5379. doi: $10.21037 /$ tcr-19-2633
24. de Santibañes M, Boccalatte L, de Santibanes E. A literature review of associating liver partition and portal vein ligation for staged hepatectomy (ALPPS): so far, so good. Updates Surg 2017;69:9-19.

25. Schnitzbauer AA, Lang SA, Goessmann H, et al. Right portal vein ligation combined with in situ splitting induces rapid left lateral liver lobe hypertrophy enabling 2-staged extended right hepatic resection in small-for-size settings. Ann Surg 2012;255:405-14.

26. Zhang Y, Zheng Y, Dong X, et al. Associating liver partition and portal vein ligation for staged hepatectomy versus conventional staged hepatectomy: a meta-analysis. Minerva Med 2018;109:141-9.

27. Chia DKA, Yeo Z, Loh SEK, et al. Greater hypertrophy can be achieved with associating liver partition with portal vein ligation for staged hepatectomy compared to conventional staged hepatectomy, but with a higher price to pay? Am J Surg 2018;215:131-7.

28. Chia DKA, Yeo Z, Loh SEK, et al. ALPPS for Hepatocellular Carcinoma Is Associated with Decreased Liver Remnant Growth. J Gastrointest Surg 2018;22:973-80.

29. Nagasue N, Yukaya H, Ogawa Y, et al. Human liver regeneration after major hepatic resection. A study of normal liver and livers with chronic hepatitis and cirrhosis. Ann Surg 1987;206:30-9.

30. Huang H, Lu X, Yang H, et al. Acute kidney injury after associating liver partition and portal vein ligation for staged hepatectomy for hepatocellular carcinoma: two case reports and a literature review. Ann Transl Med 2019;7:795.

31. Tanaka K. Modified ALPPS procedures: more safety through less invasive surgery. Langenbecks Arch Surg 2017;402:563-74. 